\title{
Viewpoint
}

\section{All that is gold does not glitter}

\author{
Nikos Daniilidis and Hartmut Häffner \\ Department of Physics, University of California, Berkeley, CA 94720-7300, USA
}

Published August 22, 2011

A microscopic model offers new insight into a pernicious source of electric field noise in ion traps.

\author{
Subject Areas: Atomic and Molecular Physics
}

\author{
A Viewpoint on: \\ Microscopic model of electric-field-noise heating in ion traps \\ A. Safavi-Naini, P. Rabl, P. F. Weck, and H. R. Sadeghpour \\ Phys. Rev. A 84, 023412 (2011) - Published August 22, 2011
}

Considering how it affects science and technology, surprisingly little is known about the electric field noise generated near the surface of metals. Now, in a theoretical paper appearing in Physical Review A, Arghavan SafaviNaini at the Massachusetts Institute of Technology in Cambridge and colleagues have uncovered a source of noise from the surfaces of metals that has been a major headache for ion trapping experiments. They argue that electrical dipoles, formed by impurity atoms adsorbed on the metal electrodes of an ion trap, cause noise of just the right strength and characteristics to explain the noise that has been observed in ion trap experiments [1. Their work provides a guideline for attacking electric field noise and could impact many fields, including surface and materials science, modern electronics, quantum information technology, and precision tests of fundamental physics.

Scientists in all of these disciplines strive to have an electrically quiet environment for their measurements. Is there, however, a fundamental lower limit for electric noise generated by an ordinary conductor, such as a piece of gold? Although electric field (or voltage) noise from the thermal motion of the freely moving electrons inside a conductor is well understood, this so-called JohnsonNyquist noise is typically not the sole source. In practice, nonequilibrium noise mechanisms, such as flicker and shot noise, often dominate device performance.

Controlling noise is particularly important for modern applications that use ion traps, such as ion-trap quantum computing. In ion traps, electrodes, usually metallic, generate electric fields that confine the ions to a small volume close by. When researchers started laser-cooling ions to the ground state of the trap, they expected that the ions would stay cold for many minutes. However, electric field noise from the trap electrodes heated up the ions within milliseconds, several orders of magnitude faster than what was expected from Johnson-Nyquist noise [2, 3]. The intensity of this unexpected noise appeared to drop with the ion's distance $d$ from the metal as $1 / d^{4}$ and the noise appeared to be thermally activated

DOI: $10.1103 /$ Physics.4.66

URL: http://link.aps.org/doi/10.1103/Physics .4.66
[4. Trying to piece the puzzle together, researchers tried a host of different metals and even tested traps with semiconducting and superconducting electrodes. Still, no clear picture of what was causing the excessive noise emerged and even initially promising models could not explain the size of the field noise 3 .

Early on, researchers guessed that the $1 / d^{4}$ scaling could be the result of a large number of uncorrelated electrical-dipole-type noise sources sitting on the metal surfaces. For example, in the so-called patch-potential model, dipolelike fields are caused by patches on the surface of a metal in which the electrons have a different work function compared to other regions on the surface. In this model, the noise arises because the patches fluctuate as impurities adsorbed on the trap electrodes diffuse around the surface, similar to the mechanism causing flicker noise in field-emission tips [3, 5]. The model seemed plausible, since even the electrode surfaces in an ultrahigh vacuum ion trap apparatus can be strongly contaminated [5]. It turned out, however, that the surface patch model predicted noise that was much lower in spectral densities than what was observed 3 .

Researchers tried to think of mechanisms other than diffusing impurities that would lead to patches and increased noise. Grain boundaries, material interfaces, and the bulk of the material itself were suspected to contribute to the noise, but a clear mechanism to explain it was lacking. One reason researchers were reluctant to abandon the patch model was that scientists in other fields were also encountering problems related to patches, although in different frequency and distance regimes. For example, scanning probe microscopy [6], the detection of Casimir forces [7], measurements of free fall of charged particles [8], and tests of general relativity [9] all encounter patch-potential effects.

In all of this work, one important aspect might have been underestimated: Impurity atoms adsorb on the surfaces and the individual adsorbed atoms form electrical dipoles (Fig. 1). The thermal motion of these adsorbed 


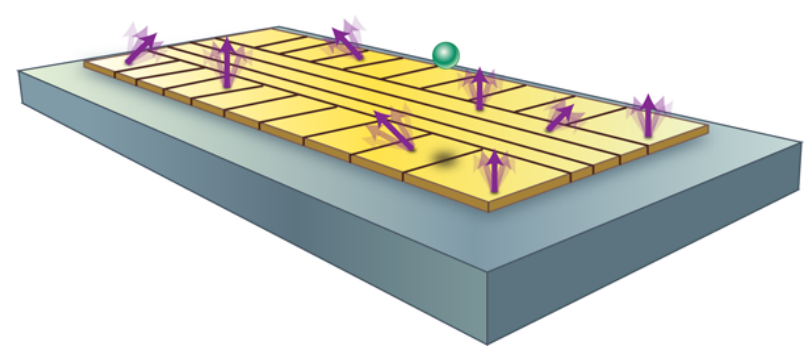

FIG. 1: What is causing trapped ions to heat up? In an ion trap, electrodes (shown here in gold) produce electric fields that confine the ion (green sphere) to a small volume. Randomly fluctuating dipoles on the surface of these electrodes generate electric field noise. The dipoles form when single atoms adsorb on the metal surface. Vibrations (phonons) in the metal cause the dipoles (purple arrows) to fluctuate. Ions trapped in the vicinity of the metal surface sense these electric field changes and heat up. (APS/Carin Cain)

atoms excites the dipoles, giving rise to electric field noise. Earlier this year, a team of experimentalists (including us) turned their attention to this mechanism and put forth a phenomenological model in which the noise arises from such fluctuating dipoles 10 . We found that reasonable parameters for the fluctuating dipoles could explain the observed noise, but our toy model lacked detail on the microscopic level and it was unclear what was activating the dipoles.

Now Safavi-Naini et al. have found an exact theory of how surface adsorbates can cause electric field noise [1. In their picture, an impurity atom adsorbed on a flat metal surface forms the analog of a van der Waals bond with the metal atoms at the surface. The adatom occupies one of many vibrational states, and since there is some charge transfer between the adatom and the metal (the bond is polar), different states give rise to electrical dipoles of different strength. It turns out that the energy spacing between different states of this multilevel dipole span an energy range in which acoustic phonons (i.e., ultrasound waves) in a typical metal exist. Thus the phonons can "kick" the adatoms, giving rise to fluctuating elementary electrical dipoles. The typical transition rates between the different dipole strengths turn out to be in the megahertz range, exactly the relevant frequency regime for trapped ions. Safavi-Naini et al. have worked out the dynamics of individual dipoles that fluctuate independently and found noise with spectral characteristics compatible with the noise measured with trapped ions. Furthermore, the power and frequency spectrum, which they calculate for the simple case of a gold surface covered with $10 \%$ of a neon monolayer, agrees with experiment. Encouragingly, the model also correctly predicts the temperature dependence that has been observed in experiments [4].
More work will undoubtedly have to follow. On the theory front, it is tempting to extend the model to include more types of surfaces, such as semiconductors, to clarify how it is related to the traditional patch potential model, and to study its implications for noise and dissipation encountered in non-ion-trap experiments [6 9]. On the experimental side, the predictions of the model can now be put to the test. The model predicts the frequency and temperature scaling of the noise, as well as its dependence on the mass and binding energies of the contaminating atoms. The first steps for testing these will be to clean trap surfaces, to prepare surfaces with different contaminants and study their noise characteristics, as well as to passivate the metal surfaces using standard surface science methods. Of course, while the situation now appears to be quite clear, one should be aware that we are dealing with real materials and be prepared for more surprises.

The work by Safavi-Naini et al. is important in at least two respects. First, it puts forward a detailed mechanism to explain the excessive electric field noise observed in ion trapping and possibly other fields. Second, the model gives experimentalists clear guidelines on how to remove at least part of the excess noise. If these efforts are successful, the work by Safavi-Naini et al. could put the glitter back into gold - or any other electrode material - and make an important step towards a scalable ion trap quantum computer.

\section{References}

[1] A. Safavi-Naini, P. Rabl, P. Weck, and H. Sadeghpour, Phys. Rev. A 84, 023412 (2011).

[2] C. Monroe, D. M. Meekhof, B. E. King, S. R. Jefferts, W. M. Itano, D. J. Wineland, and P. Gould, Phys. Rev. Lett. 75, 4011 (1995).

[3] D. J. Wineland, C. Monroe, W. M. Itano, D. Leibfried, B. King, and D. M. Meekhof, J. Res. Natl. Inst. Stand. Technol. 103, 259 (1998); Q. A. Turchette, D. Kielpinski, B. E. King, D. Leibfried, D. M. Meekhof, C. J. Myatt, M. A. Rowe, C. A. Sackett, C. S. Wood, W. M. Itano, C. Monroe, and D. J. Wineland, Phys. Rev. A 61, 063418 (2000).

[4] L. Deslauriers, S. Olmschenk, D. Stick, W. K. Hensinger, J. Sterk, and C. Monroe, Phys. Rev. Lett. 97, 103007 (2006); J. Labaziewicz, Y. Ge, D. Leibrandt, S. X. Wang, R. Shewmon, and I. L. Chuang, Phys. Rev. Lett. 101, 180602 (2008).

[5] Dustin Hite (private communication).

[6] Werner A. Hofer, Adam S. Foster, and Alexander L. Shluger, Rev. Mod. Phys. 75, 1287 (2003).

[7] W. J. Kim, A. O. Sushkov, D. A. R. Dalvit, and S. K. Lamoreaux, Phys. Rev. Lett. 103, 060401 (2009).

[8] T. W. Darling, F. Rossi, G. I. Opat, and G. F. Moorhead, Rev. Mod. Phys. 64, 237 (1992).

[9] C. W. F. Everitt et al., Phys. Rev. Lett. 106, 221101 (2011).

[10] N. Daniilidis, S. Narayanan, S. A. Möller, R. Clark, T. E. Lee, P. J. Leek, A. Wallraff, St. Schulz, F. Schmidt-Kaler, and H. Häffner, New J. Phys. 13, 013032 (2011). 


\section{About the Authors}

\section{Nikos Daniilidis}

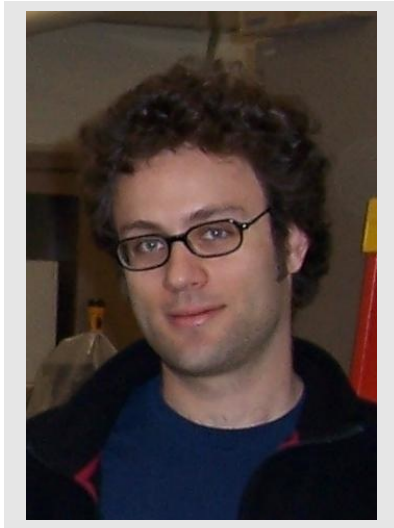

Nikos (Nikolaos) Daniilidis is a postdoctoral researcher at the University of California, Berkeley. He received his Ph.D. from Brown University and has worked as a Marie Curie fellow at the Institute for Quantum Optics and Quantum Information in Innsbruck, Austria, and as a postdoctoral researcher at ETH, Zürich. His research interests include applications of ion traps to quantum information and to hybrid quantum systems, and materials science issues related to ion trap development.

\section{Hartmut Häffner}

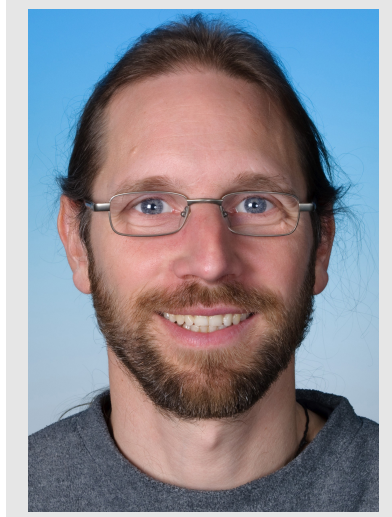

Hartmut Häffner is Assistant Professor of Physics at the University of California, Berkeley, and is also affiliated with Lawrence Berkeley National Laboratory. He received his Ph.D. in physics from the University of Mainz in Germany. He has done postdoctoral work in Mainz, Bangalore, and at NIST (Gaithersburg), and worked as a university assistant at the University of Innsbruck, Austria, as a senior scientist at the Institute for Quantum Optics and Quantum Information, Innsbruck. He has been awarded a Feodor-Lynen fellowship, a Marie Curie fellowship, a START award, and a Sloan fellowship. His research focuses on ion-trap-based quantum information, hybrid quantum systems, quantum simulations, and materials science aspects of ion traps. 